\title{
Voluntary Participation and Provision of Public Goods in Large Finite Economies*
}

\author{
Hideo Konishi $^{\dagger} \quad$ Ryusuke Shinohara ${ }^{\ddagger}$
}

August 26, 2011

\begin{abstract}
We consider a public good provision game with voluntary participation. Agents participating in the game provide a public good and pay the fees according to a mechanism (allocation rule), while nonparticipants can free-ride on the participants. We examine how the equilibrium public good provision level is affected by enlarging the population of an economy. We introduce a condition for an allocation rule, the asymptotic uniform continuity in replication (AUCR), which requires that small changes in the population must yield only small changes in the public good provision and which is satisfied by many mechanisms. We show that under AUCR, the equilibrium level of the public good converges to zero as the economy is replicated in the sense of Milleron (1972).
\end{abstract}

Keywords. public good provision, participation game, replicated economy.

JEL Classification Numbers. C72, H41.

${ }^{*}$ We thank P.J. Healy, Yukio Koriyama, Toshiji Miyakawa, Hervé Moulin, Koji Takamiya, and the two anonymous referees and the associate editor of the journal for their helpful comments. We also thank participants at Hitotsubashi COE Lectures and Workshop on Choice, Games, and Welfare (January, 2009), Public Economics Workshop at Nagoya Gakuin University (May, 2009), and Theory Workshop at Niigata University (June, 2009).

†Department of Economics, Boston College, 140 Commonwealth Avenue Chestnut Hill, MA 02467, USA. (E-mail) hideo.konishi@bc.edu

${ }^{\ddagger}$ Faculty of Economics, Shinshu University, 3-1-1, Asahi, Matsumoto, Nagano 390-8621, Japan. (E-mail) ryusukes@shinshu-u.ac.jp 


\section{Introduction}

It is well known that the provision of public goods is subject to free-riding incentives. Although Samuelson's (1954) view of this problem is pessimistic, Groves and Ledyard (1977) show that the efficient provision of public goods can be achieved in a Nash equilibrium by appropriately crafting a public goods mechanism. ${ }^{1}$ However, since the provision of pure public goods intrinsically involves non-excludability of the benefits, assuming that all agents participate in the mechanism may not be reasonable if we allow agents to decide whether or not to do so. In this paper, we will examine if agents are actually willing to participate in a public goods mechanism voluntarily. ${ }^{2}$ In particular, we are interested in how the equilibrium provision of a public good is affected by increases in the population.

Replicating a public goods economy is a difficult matter. ${ }^{3}$ In contrast to a private goods economy, if we simply replicate the number of agents then the set of Pareto efficient allocations will be modified at each replication. To avoid this issue, Milleron (1972) devised a way to duplicate a public goods economy without affecting the set of Pareto efficient allocations by modifying agents' preferences as replications proceed. In the domain of quasilinear utility preferences, by normalizing endowment at the origin, Milleron's replication goes as follows: if an agent's utility is $z+u(g)$ in the original economy then in an $r$-times replicated economy, her utility is $z+\frac{1}{r} u(g)$, where $z$ and $g$ denote private and public goods consumption, respectively.

\footnotetext{
${ }^{1}$ Although the Groves-Ledyard mechanism does not satisfy individual rationality, Hurwicz (1979) and Walker (1981) show that the Lindahl rule is implementable: the Lindahl rule is individually rational as well as efficient. Subsequently, numerous further mechanisms have been proposed to satisfy additional desirable properties. They all assume, however, that agents must participate in the mechanism: i.e., they assume that agents have no freedom to leave the mechanism.

${ }^{2}$ Saijo and Yamato (1999) considered a voluntary participation game in a divisible public goods case. They show a negative result on efficiency of public goods provision, and then characterize equilibrium participation of the mechanism that implements the Lindahl allocation rule in a symmetric Cobb-Douglas utility case. Saijo and Yamato (2010) considered a voluntary participation game in a public good economy, and show that for all agents the participation incentives in the mechanisms that implement the Lindahl rule become weaker as the number of agents in the economy increases.

${ }^{3}$ Muench (1972), Milleron (1972), and Conley (1994) discuss the difficulty of replicating a public goods economy and offer various possible methods. Milleron's notion of replication is to split endowments with replicates and adjust preferences so that agents' concerns for the private good are relative to the size of their endowments.
} 
As is easily seen, the Samuelson rule indicates the same efficient public goods provision level irrespective of the number of replications $r$.

Recently, Healy (2010) and Furusawa and Konishi (2011) show that the non-participation (free-riding) problem becomes more serious as the population increases, following Milleron's (1972) definition of a replicated economy. Although they prove that every Nash equilibrium level of a public good goes to zero as the economy is infinitely replicated, the patterns of convergence are very different from each other. Healy (2010) uses a fixed contribution rule with equilibrium participation, and shows that all agents continue to participate in the mechanism but contribute less to the public good as replications proceed. ${ }^{4}$ By contrast, Furusawa and Konishi (2011) consider the core as the public goods provision mechanism, and show that participants contribute an efficient level of public goods (for contributers) but the fraction of contributors becomes smaller and smaller as the replications proceed. In the current paper, we consider a general mechanism in the domain of quasilinear utilities, investigating the conditions on the mechanism under which all Nash equilibrium public goods provision levels converge to zero as replications proceed. We will show that our result covers both results in Healy (2010) and Furusawa and Konishi (2011) in the domain of quasi-linear preferences, despite the fact that the convergence patterns differ in these two results.

As the condition on rules of public goods provision, we propose asymptotic uniform continuity in replication (AUCR). Regarding the Milleron replications of an economy, AUCR requires that if the composition of participants are close (in a particular sense), the public goods provision levels are uniformly close in all replicated economies. We impose no condition on cost-sharing of public goods provision, and we allow for budget-surplus. Although Milleron's replication does not affect the feasibility of aggregated allocations, AUCR does not require that the public goods provision level is unaffected by replications. We show that if a public goods provision rule satisfies AUCR, then all equilibrium public goods provision levels converge to zero as an economy is replicated (Theorem 1). The class of rules that satisfy AUCR contains many popular allocation rules in mechanism design theory. The efficient allocation rules such as the Lindahl and the core allocation rules

\footnotetext{
${ }^{4}$ The fixed contribution rule assigns the same fee for each agent independent of the other agents' participation. The equilibrium participation rule is such that under this rule, all agents voluntarily participate in the public goods provision. Healy uses a domain of monotone and continuous preferences.
} 
satisfy AUCR, as does the Clarke (1971) rule. All fixed contribution rules with or without equilibrium participation also satisfy AUCR on the quasilinear preference domain. Hence, using our result, we can show that, under the mechanisms that are popular in mechanism design theory, the equilibrium level of the public good diminishes to zero as the economy is replicated, at least in the domain of quasi-linear preferences.

Our result also shows that if some positive public good is provided at a Nash equilibrium in the limit, an allocation rule has discontinuity around the Nash equilibrium level of the public good. The discontinuous allocation rule has a structure similar to the model of the provision of a discrete public good such as Palfrey and Rosenthal (1984) and Shinohara (2009).

This paper is organized as follows: in Section 2, we introduce the participation game with a public good, and in Section 3, we introduce AUCR and present the main result. Section 4 provides examples of public goods mechanisms that satisfy AUCR. Section 5 concludes the paper.

\section{The Model}

The economy contains one private good and one public good. The preference domain we consider is quasi-linear in the private good, i.e., each agent's preference is represented by a utility function $U: \mathbb{R} \times \mathbb{R}_{+} \rightarrow \mathbb{R}$ such that $U(z, g)=z+u(g)$, where $z$ and $g$ are private and public goods consumption levels, respectively, and $u: \mathbb{R}_{+} \rightarrow \mathbb{R}_{+}$is a subutility function from public goods consumption with $u(0)=0$. We normalize each agent's private good endowment to zero. That is, $-z$ denotes the contribution of an agent for public goods provision. We assume that agents have deep pockets, and that they can contribute as much as they want without being constrained by a budget condition placed by private good endowment. This assumption is standard in the domain of quasi-linear preferences (transferable utility), and is imposed to avoid unnecessary notational complications. ${ }^{5}$ Let $C: \mathbb{R}_{+} \rightarrow \mathbb{R}_{+}$ be a cost function of public goods provision with $C(0)=0$.

Let $\mathcal{U}$ and $\mathcal{C}$ be the admissible domains of utility and cost functions $u: \mathbb{R}_{+} \rightarrow \mathbb{R}_{+}$and $C: \mathbb{R}_{+} \rightarrow \mathbb{R}_{+}$. We consider the Milleron's replication of economies and we assume that if $u \in \mathcal{U}$, then $\frac{1}{r} u \in \mathcal{U}$ for all $r \in \mathbb{Z}_{++}$, where $\mathbb{Z}_{++}=\{1,2, \ldots\}$ is the set of positive integers. Later, we consider various

\footnotetext{
${ }^{5}$ This assumption tightens the problem, so all the results in the paper will be satisfied with a budget constraint.
} 
domain restrictions of $\mathcal{U}$ and $\mathcal{C}$ for each result. For our main result (and for all other results), we impose the following conditions. (i) The slope of every utility function is uniformly bounded from above: (there is $0<M<\infty$ such that $\frac{u\left(g_{1}\right)-u\left(g_{2}\right)}{g_{1}-g_{2}} \leq M$ holds for all $u \in \mathcal{U}$ and all $g_{1}>g_{2} \geq 0$ ). (ii) Cost function is nondecreasing $\left(C\left(g_{1}\right) \geq C\left(g_{2}\right)\right.$ for all $C \in \mathcal{C}$ and all $\left.g_{1}>g_{2} \geq 0\right)$ and satisfies no free lunch $(C(0)=0$ and $C(g)>0$ for all $C \in \mathcal{C}$ and all $g>0)$. In applying our result to various mechanisms, we also consider more restrictive domains of utility and/or cost functions such as differentiability, concavity, and convexity.

Let $\nu: \mathcal{U} \rightarrow \mathbb{Z}_{+}$be a population allocation that describes the population distribution of preference types, where $\mathbb{Z}_{+}=\{0,1,2, \ldots\}$ is the set of nonnegative integers. Let $\operatorname{Supp}(\nu) \equiv\{u \in \mathcal{U} \mid \nu(u)>0\}$ be the set of existing utility functions under population allocation $\nu$ and let $|S u p p(\nu)|$ be the cardinality of $\operatorname{Supp}(\nu)$ - the total population. ${ }^{6}$ Let $\mathcal{N}$ be the collection of all finite population allocations that satisfy $|\operatorname{Supp}(\nu)|<\infty$. For all $\nu \in \mathcal{N}, \nu^{\prime} \in \mathcal{N}$ is a subpopulation allocation of $\nu$ if and only if $\nu^{\prime} \leq \nu$ : i.e., $\nu^{\prime}(u) \leq \nu(u)$ for all $u \in \mathcal{U}$. We typically describe the participants of a mechanism by a subpopulation $\nu^{\prime}$ among the players $\nu$. For all $\nu \in \mathcal{N},|\nu| \equiv \sum_{u \in \operatorname{Supp}(\nu)} \nu(u)$. For all $\nu, \nu^{\prime}, \nu^{\prime \prime} \in \mathcal{N}$ such that $\nu^{\prime}$ and $\nu^{\prime \prime}$ are subpopulation allocations of $\nu$, $\left|\nu^{\prime}-\nu^{\prime \prime}\right| \equiv \sum_{u \in \operatorname{Supp}(\nu)}\left|\nu^{\prime}(u)-\nu^{\prime \prime}(u)\right|$.

An economy is a pair $(\nu, C)$ with $\nu \in \mathcal{N}$ and $C \in \mathcal{C}$. Let $\mathcal{E}$ be the collection of all economies. Below, we define allocation rules that map an economy $(\nu, C)$ and its subpopulation $\nu^{\prime} \leq \nu$ (participants of the mechanism) to public goods provision level $g$ and the cost-sharing among participants $\tau$. A (symmetric) transfer function $\tau: \mathcal{U} \rightarrow \mathbb{R}$ assigns a payment amount to each type of agent, and $\mathcal{T}$ denotes the collection of all transfer functions. An allocation rule is a function $\varphi: \mathcal{N} \times \mathcal{N} \times \mathcal{C} \rightarrow \mathcal{T} \times \mathbb{R}_{+}$such that

$$
\varphi\left[\nu^{\prime} ; \nu, C\right]=\varphi_{\mathcal{T}}\left[\nu^{\prime} ; \nu, C\right] \times \varphi_{G}\left[\nu^{\prime} ; \nu, C\right],
$$

\footnotetext{
${ }^{6}$ It is convenient for our purposes to use population allocations $\nu$ to describe an economy. In a private goods economy, an $r$-replica economy with set of (types of) consumers $N=\{1, \ldots, t, \ldots T\}$ is described by $N_{r}=\{(1,1), \ldots,(t, q), \ldots,(T, r)\}$ where $q \in\{1, \ldots, r\}$ and $(t, q)$ denotes the $q$ th type $t$ consumer in the standard notations (see, say, Wooders 1989). Here, we describe a consumer's type $t$ by her utility function $u$. With Milleron's $r$-replica of public goods economy, each type's utility function $u$ is transformed to $\frac{1}{r} u$ (see Section $3)$. So, the domain of an allocation rule $\mathcal{U}$ needs to contain all of these functions, and representing an economy by population allocation is an easy way to deal with the Milleron replications. Therefore, the population allocation approach suits our analysis.
} 
where $\nu^{\prime}$ is subpopulation allocation of $\nu\left(\nu^{\prime} \leq \nu\right)$, and $\varphi_{\mathcal{T}}\left[\nu^{\prime} ; \nu, C\right]: \mathcal{U} \rightarrow \mathbb{R}$ is a transfer function that assigns a transfer payment to each existing type of agent $u \in \mathcal{U}$ with $\nu^{\prime}(u)>0$ in economy $(\nu, C)$, and $\varphi_{G}\left[\nu^{\prime} ; \nu, C\right] \in \mathbb{R}_{+}$ assigns the amount of public goods. Note that we are assuming that if two agents have the same utility function, then their transfer payment is the same (equal treatment for each type). ${ }^{7}$ Thus, $\varphi_{\mathcal{T}}\left[\nu^{\prime} ; \nu, C\right]: \mathcal{U} \rightarrow \mathbb{R}$ satisfies (i) $\varphi_{\mathcal{T}}\left[\nu^{\prime} ; \nu, C\right](u)=0$ for all $u \notin \operatorname{Supp}\left(\nu^{\prime}\right)$ (cost is financed only by participants), and (ii)

$$
\sum_{u \in \operatorname{Supp}\left(\nu^{\prime}\right)} \nu^{\prime}(u) \times \varphi_{\mathcal{T}}\left[\nu^{\prime} ; \nu, C\right](u) \geq C\left(\varphi_{G}\left[\nu^{\prime} ; \nu, C\right]\right),
$$

(the public goods provision level is feasible in $\nu^{\prime}$ ). It follows immediately from (ii) that if a level of the public good is feasible in some subpopulation $\nu^{\prime} \leq \nu$, then it is also feasible in $\nu$.

In the economy $(\nu, C)$, an agent's type is indicated by $i \in\{1,2, \ldots|\operatorname{Supp}(\nu)|\}$, and there are $\nu\left(u_{i}\right)$ agents whose utility function is $u_{i}$. Denote a generic $u_{i^{-}}$ type agent as $i_{q}$ for $q \in\left\{1, \ldots, \nu\left(u_{i}\right)\right\}$. The set of agents in the economy is $N^{\nu} \equiv\left\{i_{q}: u_{i} \in S u p p(\nu)\right.$ and $\left.q \in\left\{1, \ldots, \nu\left(u_{i}\right)\right\}\right\}$. A participation game in $(\nu, C) \in \mathcal{E}$ with $\varphi, \Gamma(\nu, C, \varphi)$, is a list $\left(N^{\nu},\left(\{0,1\}, h_{i_{q}}\right)_{i_{q} \in N^{\nu}}\right)$, where $\{0,1\}$ is a common strategy set and $h_{i_{q}}$ is agent $i_{q}$ 's payoff function. Each agent $i_{q}$ chooses 1 (participation) or 0 (non-participation), simultaneously. Let $s: N^{\nu} \rightarrow\{0,1\}$ be a profile of the decisions. Then, the set of participants is determined. Let $\mu[s]: \mathcal{U} \rightarrow \mathbb{Z}_{+}$be such that $\mu[s]\left(u_{i}\right) \equiv\left|\left\{q \mid s_{i_{q}}=1\right\}\right|$ for all $u_{i} \in \operatorname{Supp}(\nu)$, and $\mu[s]\left(u_{j}\right)=0$ for all $u_{j} \notin \operatorname{Supp}(\nu)$. That is, $\mu[s]$ is the population distribution of participants at $s$ and $\mu[s] \in \mathcal{N}$. The payoff of $i_{q}$ at $s$ is

$$
h_{i_{q}}\left(s_{i_{q}}, s_{-i_{q}}\right)=\left\{\begin{array}{ll}
u_{i}\left(\varphi_{G}[\mu[s] ; \nu, C]\right)-\varphi_{\mathcal{T}}[\mu[s] ; \nu, C]\left(u_{i}\right) & \text { if } s_{i_{q}}=1 \\
u_{i}\left(\varphi_{G}[\mu[s] ; \nu, C]\right) & \text { if } s_{i_{q}}=0
\end{array} .\right.
$$

A (pure-strategy) Nash equilibrium $s$ of a participation game $\Gamma(\nu, C, \varphi)$ is such that for all $i_{q} \in N^{\nu}, h_{i_{q}}\left(s_{i_{q}}, s_{-i_{q}}\right) \geq h_{i_{q}}\left(s_{i_{q}}^{\prime}, s_{-i_{q}}\right)$ holds, where $s_{i_{q}}^{\prime} \neq s_{i_{q}}$. We denote the set of Nash equilibria by $N E(\Gamma(\nu, C, \varphi))$. For $s$ and $u_{i}$ such that $s_{i_{q}}=1$ for some $q \in\left\{1, \ldots\left|\nu\left(u_{i}\right)\right|\right\}$, let $\mu_{(i,-1)}[s]: \mathcal{U} \rightarrow \mathbb{Z}_{+}$be such that

\footnotetext{
${ }^{7}$ It would be possible to drop this assumption without changing our result, although it is quite natural to impose "equal treatment" for each type. If this assumption is dropped, then an agent who is asked to pay more than others in the same type has a stronger incentive to free-ride. So, equal-treatment is the most effective way to maximize agents' participation in the mechanism.
} 
$\mu_{(i,-1)}[s]\left(u_{i}\right)=\mu[s]\left(u_{i}\right)-1$ and $\mu_{(i,-1)}[s](u)=\mu[s](u)$ for all $u \neq u_{i}$. For $s$ and $u_{i}$ such that $s_{i_{q}}=0$ for some $q \in\left\{1, \ldots\left|\nu\left(u_{i}\right)\right|\right\}$, let $\mu_{(i,+1)}[s]: \mathcal{U} \rightarrow \mathbb{Z}_{+}$ be such that $\mu_{(i,+1)}[s]\left(u_{i}\right)=\mu[s]\left(u_{i}\right)+1$ and $\mu_{(i,+1)}[s](u) \stackrel{(=)}{=} \mu[s](u)$ for all $u \neq u_{i}$. We can rewrite the definition of Nash equilibrium: a strategy profile $s$ is a Nash equilibrium if and only if

(i) for all $i_{q} \in N^{\nu}$ such that $s_{i_{q}}=1$ and $u_{i} \in \operatorname{Supp}(\nu)$,

$$
u_{i}\left(\varphi_{G}[\mu[s] ; \nu, C]\right)-\varphi_{\mathcal{T}}[\mu[s] ; \nu, C]\left(u_{i}\right) \geq u_{i}\left(\varphi_{G}\left[\mu_{(i,-1)}[s] ; \nu, C\right]\right), \text { and }
$$

(ii) for all $i_{q} \in N^{\nu}$ such that $s_{i_{q}}=0$ and $u_{i} \in \operatorname{Supp}(\nu)$,

$$
u_{i}\left(\varphi_{G}[\mu[s] ; \nu, C]\right) \geq u_{i}\left(\varphi_{G}\left[\mu_{(i,+1)}[s] ; \nu, C\right]\right)-\varphi_{\mathcal{T}}\left[\mu_{(i,+1)}[s] ; \nu, C\right]\left(u_{i}\right) .
$$

Thus, by using (i), we have the following lemma, which will be useful in obtaining our main result in the next section.

Lemma 1 A necessary condition for a strategy profile $s$ to be a Nash equilibrium when $|\mu[s]| \neq 0$ is

$$
\sum_{u_{i} \in \operatorname{Supp}(\nu)} \mu[s]\left(u_{i}\right)\left[u_{i}\left(\varphi_{G}[\mu[s] ; \nu, C]\right)-u_{i}\left(\varphi_{G}\left[\mu_{(i,-1)}[s] ; \nu, C\right]\right)\right] \geq C\left(\varphi_{G}[\mu[s] ; \nu, C]\right) .
$$

\section{Asymptotic Uniform Continuity in Repli- cations}

This section introduces our main condition, asymptotic uniform continuity in replications, and proves the main result of the paper. First, we define a replica of our economy. For all $u \in \mathcal{U}$ and $r \in \mathbb{Z}_{++}$, let $u^{r} \in \mathcal{U}$ be such that $u^{r}(g)=\frac{1}{r} u(g)$ for all $g \in \mathbb{R}_{+}$. For all $(\nu, C) \in \mathcal{E}$, let $\left(\nu^{r}, C\right) \in \mathcal{E}$ be $r$-replication of $(\nu, C)$ such that $\nu^{r}\left(u^{r}\right)=r \nu(u)$ for all $u \in \mathcal{U}$. This way of replicating a public goods economy was first defined by Milleron (1972). ${ }^{8}$ It basically divides each agent into smaller pieces as replication proceeds. One of the merits of Milleron's notion of replication is that the efficient level of public goods provision is invariant with replications. Note, however, that $\operatorname{Supp}(\nu) \neq \operatorname{Supp}\left(\nu^{r}\right)$ for all $r>1$, since preferences are altered after

\footnotetext{
${ }^{8}$ This simple definition is an adaptation from Milleron (1972), but in quasi-linear economies.
} 
replication. We impose the following condition only on public goods provision levels (not on transfer functions).

AUCR - asymptotic uniform continuity in replications: For all $(\nu, C) \in \mathcal{E}$, and all $\epsilon>0$, there exist $\delta>0$ and $\bar{r} \in \mathbb{Z}_{++}$such that for all $r \geq \bar{r}$ and all $\widetilde{\nu}, \bar{\nu} \in \mathcal{N}$ such that $\widetilde{\nu} \leq \bar{\nu} \leq \nu^{r}$ and $\frac{|\widetilde{\nu}-\bar{\nu}|}{\left|\nu^{r}\right|} \leq \delta$,

$$
\left|\varphi_{G}\left[\widetilde{\nu} ; \nu^{r}, C\right]-\varphi_{G}\left[\bar{\nu} ; \nu^{r}, C\right]\right|<\epsilon
$$

holds.

Here is an interpretation of AUCR. It requires continuity of public goods provision in a population uniformly for large enough numbers of replications of the economy. Let $e \in \mathcal{E}$. For all $\widetilde{\nu}, \bar{\nu} \in \mathcal{N}$ such that $\widetilde{\nu}, \bar{\nu} \leq \nu, \frac{|\widetilde{\nu}-\bar{\nu}|}{|\nu|}$ represents the ratio of the difference in populations between $\widetilde{\nu}$ and $\bar{\nu}$ based on the whole economy. For any two subpopulations, if the ratio is sufficiently small, then the levels of the public good at the subpopulations are close. Roughly speaking, AUCR imposes the condition that if the population compositions are close, then the levels of the public good are also close.

First, note that this condition does not require that the public goods provision level stays intact for all replications. That is, we do not demand $\varphi_{G}\left[\nu^{\prime} ; \nu, C\right]=\varphi_{G}\left[r \nu^{\prime} ; \nu^{r}, C\right]$ for $r=2,3, \ldots$, where $r \nu^{\prime}$ is the $r$-replication of $\nu^{\prime}$. Second, requiring "uniform" continuity is important: if "there exists $\delta>0$ such that for all $r \in \mathbb{Z}_{++}$and all ..." is replaced by "for all $r \geq \bar{r} \in \mathbb{Z}_{++}$ there exists $\delta>0$ such that for all ..." then the statement of AUCR does not impose any restriction. This is because for all $\epsilon>0$, we can pick $\delta>0$ small enough to let $\frac{|\widetilde{\nu}-\bar{\nu}|}{\left|\nu^{r}\right|} \leq \delta$ imply $\widetilde{\nu}=\bar{\nu}$, which automatically guarantees $\varphi_{G}\left[\widetilde{\nu} ; \nu^{r}, C\right]=\varphi_{G}\left[\bar{\nu} ; \nu^{r}, C\right]$. Third, $\varphi_{G}$ is not required to be continuous in similarity of preferences (for example, in Hausdorff distance). Our continuity is only in population composition, so we can allow rules that assign very different public goods provision levels when agents' preferences change slightly. Fourth, this condition is completely silent about cost sharing of public goods. This somewhat innocuous-looking technical condition plays a central role in the subsequent analysis. ${ }^{9}$

\footnotetext{
${ }^{9}$ Small group effectiveness (Wooders, 1983, 2008) is a condition for a cooperative game, which requires that if a relatively small population deviates and changes a coalition structure, then the worth of coalitions slightly changes. Continuity with respect to attributes (Allouch and Wooders, 2008) is a condition under which a small change in consumers' attributes changes consumers' utilities and the initial endowment of a private good, slightly.
} 
The set of agents in a replicated economy $\left(\nu^{r}, C\right)$, where $r \in \mathbb{Z}_{++}$and $(\nu, C) \in \mathcal{E}$, is as follows. There are $r \nu\left(u_{i}\right)$ agents whose utility functions are $u_{i}^{r}$. Denote a generic $u_{i}^{r}$-type agent as $i_{q}^{r}$ for $q \in\left\{1, \ldots, r \nu\left(u_{i}\right)\right\}$. For a strategy profile $s_{r}: N^{\nu^{r}} \rightarrow\{0,1\}$, the population distribution of contributors is $\mu\left[s_{r}\right] \in \mathcal{N}$ such that $u_{i}^{r} \in \mathcal{U}, \mu\left[s_{r}\right]\left(u_{i}^{r}\right) \equiv\left|\left\{q \mid s_{i_{q}^{r}}=1\right\}\right|$ if $u_{i}^{r} \in \operatorname{Supp}\left(\nu^{r}\right)$ and $\mu[s](u) \equiv 0$ if $u \notin S u p p\left(\nu^{r}\right)$. As before, for all $u_{i}^{r} \in \mathcal{U}$ and $s_{r}: N^{\nu^{r}} \rightarrow$ $\{0,1\}$, let $\mu_{\left(i^{r},-1\right)}\left[s_{r}\right]: \mathcal{U} \rightarrow \mathbb{Z}_{+}$be such that $\mu_{\left(i^{r},-1\right)}\left[s_{r}\right]\left(u_{i}^{r}\right)=\mu\left[s_{r}\right]\left(u_{i}^{r}\right)-1$ and $\mu_{\left(i^{r},-1\right)}\left[s_{r}\right](u)=\mu\left[s_{r}\right](u)$ for all $u \neq u_{i}^{r}$. The set of Nash equilibria in $\Gamma\left(\nu^{r}, C, \varphi\right)$ is denoted by $N E\left(\Gamma\left(\nu^{r}, C, \varphi\right)\right)$.

The following is the key result that will lead us to our main theorem.

Lemma 2 The slope of the utility function is uniformly bounded from above, and the cost function is nondecreasing and satisfies no free lunch. Suppose that $\varphi_{G}$ satisfies AUCR. Then, for any positive amount of public good $g>0$, there is a $\bar{r} \in \mathbb{R}_{++}$such that, for any $r \geq \bar{r}$, no Nash equilibrium of $\Gamma\left(\nu^{r}, C, \varphi\right)$ exists in which $g$ or more units of the public good are provided.

Theorem 1 The slope of the utility function is uniformly bounded from above, and the cost function is nondecreasing and satisfies no free lunch. Suppose that $\varphi_{G}$ satisfies AUCR. Then, all Nash equilibrium public goods provision levels converge to zero as economy $(\nu, C)$ is replicated (i.e., $\lim _{r \rightarrow \infty} \varphi_{G}\left[\mu\left[s_{r}\right] ; n^{r}, C\right]=$ 0 for all Nash equilibrium sequence $\left\{s_{r}\right\}_{r=1}^{\infty}$ such that $s_{r} \in N E\left(\Gamma\left(\nu^{r}, C, \varphi\right)\right)$ for all $r=1,2, \ldots)$.

Note that if condition AUCR is guaranteed around the equilibrium provision of a public good, the convergence result can be established. For example, consider a rule that is continuous around the equilibrium levels but discontinuous at very high provision levels where the individual rationality condition is not satisfied for anybody; clearly, such a provision level cannot be achieved as a Nash equilibrium. Although this rule has discontinuity, all sequences converge to zero under this rule. Therefore, AUCR is not a necessary condition for convergence.

Conversely, the discontinuity around a Nash equilibrium may allow that a positive level of a public good is provided at an equilibrium even if the

At first glance, these conditions may appear to be similar to AUCR in the sense that a small change in one dimension induces a small change in another dimension. However, AUCR is a continuity condition on public good provision level, not on utility levels. 
economy is replicated. In the conclusion, we will provide a simple example of this.

Note that utility functions do not need to be concave, continuous (jumping down is allowed), or differentiable. Our result holds as long as all utility functions $u$ have their slopes uniformly bounded above by a finite number. The following example shows that if the slope of the utility function is not uniformly bounded above, there may be a Nash equilibrium sequence that does not converge to zero, even if $\varphi_{G}$ satisfies AUCR.

Example 1 Consider a one-person economy $\left\{u_{i}\right\}=\operatorname{Supp}(\nu)$ and $|\nu|=1$ such that $u_{i}(g)=2$ if $g \geq 1$, and $u_{i}(g)=0$ otherwise, and $C(g)=g$. For all $(\nu, C) \in \mathcal{E}$, all $r \in \mathbb{Z}_{++}$, and all $\widetilde{\nu} \leq \nu^{r}$, let $\varphi_{G}\left[\widetilde{\nu} ; \nu^{r}, C\right]=C^{-1}\left(\frac{|\widetilde{\nu}|}{r|\nu|}\right)$. This $\varphi_{G}$ satisfies AUCR. ${ }^{10}$ Note that $u_{i}(g)$ jumps up at $g=1$, and the condition of uniformly bounded slopes of $u$ is violated. ${ }^{11}$ Suppose that $\varphi_{\mathcal{T}}$ satisfies budget balance. Then, for all $r \in \mathbb{Z}_{++}$, there is a Nash equilibrium in which all agents participate and one unit of the public good is provided. Therefore, there is a sequence of Nash equilibria whose level of the public good does not converge to zero.

Note also that we do not impose any condition on $C$, except that it is a nondecreasing function with $C(0)=0$ and $C(g)>0$ for $g>0$.

\section{Examples of AUCR Allocation Rules}

We present allocation rules that satisfy AUCR. A voluntary participation game with a perfectly divisible public good has been studied by several authors such as Saijo and Yamato (1999, 2010), Shinohara (2010), Furusawa and Konishi (2011), and Healy (2010). As we will see below, many allocation rules, which have been studied in these papers, satisfy AUCR. Hence, every

\footnotetext{
${ }^{10}$ We show that $\phi_{G}$ satisfies AUCR. Let $\epsilon>0$. Let $k \in \mathbb{Z}_{++}$. For all $r \in \mathbb{Z}_{++}$, let $K^{r} \equiv\left\{(\widetilde{\nu}, \bar{\nu}) \mid \bar{\nu} \leq \widetilde{\nu} \leq \nu^{r}\right.$ and $\left.|\widetilde{\nu}-\bar{\nu}| \leq k\right\}$. For all $(\widetilde{\nu}, \bar{\nu}) \in K^{r}, \mid \phi_{G}\left[\widetilde{\nu} ; \nu^{r}, C\right]-$ $\phi_{G}\left[\bar{\nu} ; \nu^{r}, C\right] \mid=\frac{|\widetilde{\nu}-\bar{\nu}|}{r|\nu|} \leq \frac{k}{r|\nu|}$. Clearly, there exists $\bar{r} \in \mathbb{Z}_{++}$such that for all $r \geq \bar{r}, \frac{k}{r|\nu|}<\epsilon$. Set $\delta \equiv \frac{k}{\bar{r}|\nu|}$. Then, for all $r \geq \bar{r}$ and all $\widetilde{\nu}, \bar{\nu}$ such that $\bar{\nu} \leq \widetilde{\nu} \leq \nu^{r}$ and $\frac{|\widetilde{\nu}-\bar{\nu}|}{\left|\nu^{r}\right|} \leq \delta$, $\left|\phi_{G}\left[\widetilde{\nu} ; \nu^{r}, C\right]-\phi_{G}\left[\bar{\nu} ; \nu^{r}, C\right]\right|<\epsilon$.

${ }^{11}$ We can obtain the same result by using a continuous function $\widetilde{u}(g)$ that is sufficiently close to $u(g)$ such that $\lim _{g \uparrow 1} \widetilde{u}^{\prime}(g)=\infty$.
} 
Nash equilibrium level of public goods converges to zero as the number of replications gets large. We start with efficient provision rule of a public good.

Efficient provision rules of a public good with budget feasibility. An efficient provision rule of a public good with budget feasibility, which is denoted by $\varphi^{E}=\left(\varphi_{G}^{E}, \varphi_{\mathcal{T}}^{E}\right)$, is defined as follows: for all $(\nu, C)$ and all $\widetilde{\nu} \leq \nu$,

$$
\varphi_{G}^{E}[\widetilde{\nu} ; \nu, C] \in \arg \max _{g \geq 0} \sum_{u \in \operatorname{Supp}(\widetilde{\nu})} \widetilde{\nu}(u) u(g)-C(g) .
$$

Rule $\varphi_{G}^{E}$ assigns a public good at a level that maximizes the surplus of participants and the sum of the payments from the participants covers its production cost. If $\varphi^{E}$ satisfies the budget feasibility condition with equality, then it is called an efficient allocation rule. The Lindahl (ratio) and the core allocation rules are examples of this.

A strategy-proof mechanism is also in this class. The Clarke mechanism, introduced by Clarke (1971), is one example. The outcome obtained at dominant strategy equilibrium of the Clarke mechanism is representable by $\varphi^{\text {Clarke }}=\left(\varphi_{G}^{\text {Clarke }}, \varphi_{\mathcal{T}}^{\text {Clarke }}\right)$ in the following way: for all $(\nu, C)$ and for all $\widetilde{\nu} \leq \nu, \varphi_{G}^{\text {Clarke }}[\widetilde{\nu} ; \nu, C]=\varphi_{G}^{E}[\widetilde{\nu} ; \nu, C]$ and for all $u_{i} \in \operatorname{Supp}(\widetilde{\nu})$,

$$
\begin{aligned}
\varphi_{\mathcal{T}}^{\text {Clarke }}[\widetilde{\nu} ; \nu, C]\left(u_{i}\right) & =\frac{C\left(\varphi_{G}^{\text {Clarke }}[\widetilde{\nu} ; \nu, C]\right)}{|\widetilde{\nu}|} \\
& +\max _{g \geq 0}\left(\sum_{u \in \operatorname{Supp}(\nu)} \widetilde{\nu}_{(i,-1)}(u) u(g)-\frac{(|\widetilde{\nu}|-1) C(g)}{|\widetilde{\nu}|}\right) \\
& -\left(\sum_{u \in \operatorname{Supp}(\nu)} \widetilde{\nu}_{(i,-1)}(u) u\left(\varphi_{G}^{E}[\widetilde{\nu} ; \nu, C]\right)-\frac{(|\widetilde{\nu}|-1) C\left(\varphi_{G}^{E}[\widetilde{\nu} ; \nu, C]\right)}{|\widetilde{\nu}|}\right),
\end{aligned}
$$

where $\widetilde{\nu}_{(i,-1)}\left(u_{i}\right)=\widetilde{\nu}\left(u_{i}\right)-1$ and $\widetilde{\nu}_{(i,-1)}(u)=\widetilde{\nu}(u)$ for all $u \in \operatorname{Supp}(\nu) \backslash\left\{u_{i}\right\}$. The Clarke rule satisfies budget feasibility. The class of Groves mechanisms contains the Clarke mechanism. Not all Groves mechanisms are budget feasible. If a Groves mechanism satisfies budget feasibility, then it is an efficient provision rule with budget feasibility.

In the following, we prove that $\varphi_{G}^{E}$ satisfies AUCR in the standard domain of public good economies. We will assume that $u$ and $C$ are continuously differentiable only for simplicity, and we impose $\lim _{g \rightarrow \infty} C^{\prime}(g)=\infty$ or $\lim _{g \rightarrow \infty} u^{\prime}(g)=0$ to ensure the existence of an efficient allocation for every economy. 
Proposition 1 Let the domain of economies be such that (i) the slope of $u$ is uniformly bounded from above, (ii) $u$ is nondecreasing and concave, (iii) $C$ is nondecreasing and weakly convex, and (iv) $u$ and $C$ are continuously differentiable with $\lim _{g \rightarrow \infty} C^{\prime}(g)=\infty$ or $\lim _{g \rightarrow \infty} u^{\prime}(g)=0$. Then, rule $\varphi_{G}^{E}$ satisfies AUCR.

The following corollary is immediate from Theorem 1.

Corollary 1 Let the domain of economies be such that (i) the slope of $u$ is uniformly bounded above by $M<\infty$, (ii) $u$ is increasing and concave, (iii) $C$ is increasing and weakly convex, and (iv) $u$ and $C$ are continuously differentiable with $\lim _{g \rightarrow \infty} C^{\prime}(g)=\infty$ or $\lim _{g \rightarrow \infty} u^{\prime}(g)=0$. Then, in the participation game under $\varphi^{E}$, every sequence of public goods levels at Nash equilibria converges to zero as an economy is replicated.

Note that our domain restriction is standard and mild. The key to the above result is that as replications continue, each agent's marginal utility becomes very small, and her participation decision has a very small impact on the level of public good. The following example illustrates the importance of domain restriction for concave utility functions. Without the domain restriction, there can be multiple efficient public goods provision levels for an economy.

Example 2 Consider the following one-person economy $\left\{u_{i}\right\}=\operatorname{Supp}(\nu)$ and $|\nu|=1$ such that

$$
u_{i}(g)= \begin{cases}-g^{3}+2 g^{2} & \text { for } g \leq \frac{4}{3} \\ \frac{32}{27} & \text { for } g>\frac{4}{3}\end{cases}
$$

and

$$
C(g)=g \text {. }
$$

Let $\varphi_{G}^{E}\left[\widetilde{\nu} ; \nu^{r}, C\right]=1$ if $\widetilde{\nu}=\nu^{r}$, and $\varphi_{G}^{E}\left[\widetilde{\nu} ; \nu^{r}, C\right]=0$, otherwise. This is an efficient rule $\left(u_{i}(g)-C(g) \leq 0\right.$ for all $g \geq 0$ with equalities only at $\left.g=0,1\right)$. In this example, concavity of $u$ is violated. All other conditions are satisfied. Clearly AUCR is violated here. Indeed, $\varphi^{E}\left(\nu^{r} ; \nu^{r}, C\right)=1$ for all $r$, and $g=1$ is a Nash equilibrium outcome for all $r$.

Fixed contribution rules with budget balance. A fixed contribution rule with budget balance $\varphi^{F}=\left(\varphi_{G}^{F}, \varphi_{T}^{F}\right)$ is such that $(\mathrm{i}) \varphi_{\mathcal{T}}^{F}[\widetilde{\nu} ; \nu, C](u)=$ 
$\varphi_{\mathcal{T}}^{F}[\bar{\nu} ; \nu, C](u)$ for all $(\nu, C)$, all $\widetilde{\nu}, \bar{\nu} \leq \nu$, and all $u$ with $\widetilde{\nu}(u), \bar{\nu}(u)>0$ and (ii) $C\left(\varphi_{G}^{F}\left[\nu^{\prime} ; \nu, C\right]\right)=\sum_{u \in \operatorname{Supp}\left(\nu^{\prime}\right)} \nu^{\prime}(u) \varphi_{\mathcal{T}}^{F}\left[\nu^{\prime} ; \nu, C\right](u)$ for all $(\nu, C)$ and all $\nu^{\prime} \leq \nu$.

Condition (i) of the fixed contribution rule with budget balance means that the fee of every agent is fixed and (ii) means that a public good is provided in a budget-balancing way. Under this rule, each agent always pays the same amount of a private good for a contribution. The public good is provided by using the total amount of contributions from the agents who contributed. The agents who do not pay the fee can free-ride on others for the public good. Healy (2010) studies a voluntary participation game under this rule and analyzes the situation when all agents voluntarily participate in the mechanism (equilibrium participation).

In Proposition 2, we show that under the fixed contribution rule, AUCR is satisfied. Note that this proposition can be applied to an economy with non-convex cost function. We used only uniform boundedness of $C$, not convexity.

Proposition 2 Let the domain of economies be such that (i) the slope of $u$ is uniformly bounded from above, (ii) $u$ is nondecreasing, and (iii) $C(0)=0$ and the slope of $C$ is uniformly bounded from below by $\underline{c}>0\left(\frac{C\left(g_{1}\right)-C\left(g_{2}\right)}{g_{1}-g_{2}} \geq \underline{c}\right.$ for all $\left.g_{1}>g_{2} \geq 0\right)$. Moreover, suppose that for all $\nu \in \mathcal{N}$ and $u_{i} \in S u p p(\nu)$, there exists $\bar{G}(\nu)$ such that $\varphi_{G}^{F}\left[\nu^{r} ; \nu^{r}, C\right]<\bar{G}(\nu)$ for all $r$. Then, $\varphi_{G}^{F}$ satisfies $A U C R$.

For the above proposition to hold, it is important to have the upper bound for the public goods provision level: for all $\nu \in \mathcal{N}$ and $u_{i} \in \operatorname{Supp}(\nu)$, there exists $\bar{G}(\nu)$ such that $\varphi_{G}^{F}\left[\nu^{r} ; \nu^{r}, C\right]<\bar{G}(\nu)$ for all $r$. This condition is reasonable, if we require that $\varphi^{F}$ achieves an individually rational allocation for all economies (agents would never participate in the mechanism unless individual rationality is satisfied). ${ }^{12}$ In the following example, the individually rational public goods provision levels are not bounded from above.

Example 3 Consider the following one person economy $\operatorname{Supp}(\nu)=\left\{u_{i}\right\}$ and

\footnotetext{
${ }^{12}$ For example. if each agent's contribution is restricted by a budget constraint $\left(\frac{\omega_{i}}{r} \geq\right.$ $\varphi_{\mathcal{T}}\left[\nu^{\prime} ; \nu^{r}, C\right]\left(u_{i}^{r}\right)$ for all $r \in \mathbb{Z}_{++}$and all $\left.\nu^{\prime} \leq \nu^{r}\right)$, then there is always $\bar{G}(\nu)$. Thus, the following pathological example is derived only under the deep pocket assumption that is unrealistic though it is a standard assumption in a quasi-linear preference setting.
} 
$\nu\left(u_{i}\right)=1$ such that

$$
u_{i}(g)=g+\left(1-\frac{1}{g+1}\right)
$$

and

$$
C(g)=g .
$$

In this example, $u_{i}(g)$ is strictly concave and $C(g)$ is weakly convex. Now, consider $\varphi_{G}^{F}\left[\nu^{r} ; \nu^{r}, C\right]=r$ for all $r$. Then, $\varphi_{G}^{F}\left[\nu_{\left(i^{r},-1\right)}^{r} ; \nu^{r}, C\right]=r-1$ for all $r$, where $\nu_{\left(i^{r},-1\right)}^{r}$ is such that $\left|\nu_{\left(i^{r},-1\right)}^{r}\right|=r-1$; hence, AUCR is violated.

Note that there is no efficient allocation in the above public goods economy, which is why there is no upper bound for individually rational allocation (in fact, in this particular example, there is no efficient public goods provision level - it goes to infinity). If we ignore such a pathological case, we have the following result.

Corollary 2 Let the domain of economies be such that (i) the slope of $u$ is uniformly bounded from above, (ii) $u$ is nondecreasing, and (iii) $C(0)=0$ and the slope of $C$ is uniformly bounded below by $\underline{c}>0\left(\frac{C\left(g_{1}\right)-C\left(g_{2}\right)}{g_{1}-g_{2}} \geq \underline{c}\right.$ for all $\left.g_{1}>g_{2} \geq 0\right)$. Suppose that for all $\nu \in \mathcal{N}$ and all $u_{i} \in S u p p(\nu)$, there exists $\bar{G}(\nu)$ such that $\varphi_{G}^{F}\left[\nu^{r} ; \nu^{r}, C\right]<\bar{G}(\nu)$ for all $r$. In a voluntary participation game under $\varphi^{F}$, every sequence of the public goods level at Nash equilibria diminishes to zero as the economy is replicated.

Healy (2010) treats a domain of continuous and monotone preferences; thus, his domain is not necessarily quasi-linear. He focuses on a class of allocation rules that satisfy equilibrium participation (EP), which guarantees that all agents in the economy voluntarily choose to contribute the fee. When agents' preferences are quasi-linear, an allocation rule $\varphi=\left(\varphi_{G}, \varphi_{T}\right)$ satisfies EP if $u_{i}\left(\varphi_{G}[\nu ; \nu, C]\right)-\varphi_{\mathcal{T}}[\nu ; \nu, C]\left(u_{i}\right) \geq u_{i}\left(\varphi_{G}\left[\nu_{(i,-1)} ; \nu, C\right]\right)$ for all $(\nu, C)$ and all $u_{i} \in \operatorname{Supp}(\nu)$, where $\nu_{(i,-1)}\left(u_{i}\right)=\nu\left(u_{i}\right)-1$ and $\nu_{(i,-1)}(u)=\nu(u)$ for all $u \in \operatorname{Supp}(\nu) \backslash\left\{u_{i}\right\}$. He shows that if the fixed contribution rule with budget balance satisfies EP, then every sequence of Nash equilibrium levels of a public good goes to zero as the economy is replicated following the definition by Milleron (1972). However, EP is rather strong: for example, the fixed contribution rule with efficiency does not satisfy EP. ${ }^{13}$ With efficiency, the

\footnotetext{
${ }^{13}$ In the fixed contribution rule with efficiency, the fixed fee is determined such that if all agents in the economy participate in the public good provision, the efficient level of the public good is provided.
} 
fee of an agent is relatively high, and given that many agents participate, the other agents do not gain by joining in the provision. Although Corollary 2 is a result on the quasi-linear preference domain, EP is not assumed. Our convergence result is proven under all fixed contribution rules with budget balance.

A voluntary contribution rule. A voluntary contribution rule assigns a Nash equilibrium outcome of a voluntary contribution game, which is introduced by Bergstrom et al. (1986), for all (sub)economies. Let $(\nu, C) \in \mathcal{E}$ be an economy. The voluntary contribution game is defined as follows. Each agent in the economy simultaneously decides how many private goods he/she contributes to public goods provision. The public good is provided by the technology represented by $C$ in such a way that the cost of the public good is equal to the sum of the contributions. Formally, $X_{i}=\mathbb{R}_{+}$is the strategy set of $i \in N^{\nu}$. For each $\mathbf{x} \in \prod_{j \in N^{\nu}} X_{j}, g$ units of the public good are provided, where $C(g)=\sum_{j \in N^{\nu}} x_{j}$. The payoff of $i \in N^{\nu}$ when $\mathbf{x} \in \prod_{j \in N} X_{j}$ is selected is $u_{i}(g)-x_{i}$, where $C(g)=\sum_{j \in N^{\nu}} x_{j}{ }^{14}$

Lemma 3 Let the domain of economies be such that (i) the slope of $u$ is uniformly bounded from above, (ii) $u$ is continuously differentiable, increasing, and weakly concave, (iii) $\lim _{g \rightarrow \infty} u^{\prime}(g)=0$, and (iv) $C(0)=0$ and $C$ is continuously differentiable, increasing, and convex. The set of Nash equilibria in the voluntary contribution game is

$$
\left\{\mathbf{x} \in \prod_{j \in N^{\nu}} X_{j} \mid x_{j}=0 \text { for all } j \notin N_{\text {max }}^{\nu} \text { and } g_{\nu}^{\max }=C^{-1}\left(\sum_{j \in N_{\text {max }}^{\nu}} x_{j}\right)\right\} \text {, }
$$

where $g_{i}^{*} \in \arg \max _{g \geq 0} u_{i}(g)-C(g)$ for all $i \in N^{\nu}, g_{\nu}^{\max } \equiv \max _{i \in N^{\nu}} g_{i}^{*}$, and $N_{\text {max }}^{\nu} \equiv\left\{i \in N^{\nu} \mid g_{i}^{*}=g_{\nu}^{\max }\right\}$.

We can easily confirm that the Nash equilibrium in the voluntary contribution game is not necessarily unique. We focus on the symmetric Nash equilibrium, in which agents who have the same $u$ pay the same contribution. Using the symmetric equilibrium, we construct the voluntary contribution rule with symmetry.

For all $(\nu, C) \in \mathcal{E}$ and all $\widetilde{\nu} \leq \nu$, the voluntary contribution rule with

\footnotetext{
${ }^{14} \mathrm{~A}$ similar game and notations can be defined for all subpopulation $\widetilde{\nu} \leq \nu$, analogously.
} 
symmetry $\varphi^{V C}=\left(\varphi_{G}^{V C}, \varphi_{\mathcal{T}}^{V C}\right)$ assigns the following allocation:

$$
\begin{aligned}
& \varphi_{G}^{V C}[\widetilde{\nu} ; \nu, C]=g_{\widetilde{\nu}}^{\max }, \\
& \varphi_{\mathcal{T}}^{V C}[\widetilde{\nu} ; \nu, C]\left(u_{i}\right)= \begin{cases}\frac{C\left(g_{\widetilde{\nu}}^{\max }\right)}{\widetilde{\nu}\left(u_{i}\right)} & \text { if } g_{i}^{*}=g_{\nu^{\max }} \\
0 & \text { otherwise. }\end{cases}
\end{aligned}
$$

Under $\varphi^{V C}$, the level of the public good is determined by $g_{\widetilde{\nu}}^{\max }$, which is the greatest amount of the public good that agents in $(\widetilde{\nu}, C)$ can provide individually (recall we assume quasi-linear utility). The cost of the public good is shared by participants with $u \in \arg \max _{u \in \operatorname{Supp}(\widetilde{\nu})} g_{u}^{*}$ evenly. We show that $\varphi_{G}^{V C}$ satisfies AUCR.

Proposition 3 Let the domain of economies be such that (i) the slope of $u$ is uniformly bounded from above, (ii) $u$ is continuously differentiable, increasing, and weakly concave, (iii) $\lim _{g \rightarrow \infty} u^{\prime}(g)=0$, and (iv) $C(0)=0$ and $C$ is continuously differentiable, increasing, and convex. Then, $\varphi_{G}^{V C}$ satisfies $A U C R$.

The following corollary is an immediate implication of Theorem 1.

Corollary 3 Let the domain of economies be such that (i) the slope of $u$ is uniformly bounded from above, (ii) $u$ is continuously differentiable, increasing, and weakly concave, (iii) $\lim _{g \rightarrow \infty} u^{\prime}(g)=0$, and (iv) $C(0)=0$ and $C$ is continuously differentiable, increasing, and convex. In a participation game

with $\varphi^{V C}$, every sequence of public good levels at Nash equilibria diminishes to zero as the economy is replicated.

\section{Concluding Remarks}

This paper has addressed the nature and significance of the free-riding problem in a voluntary participation game. We examine the allocation rules underwhich the Nash equilibrium level of a public good converges to zero as agents in the economy are replicated to large numbers. We introduce a continuity concept for a public goods provision rule, AUCR. If a public goods provision rule satisfies AUCR, then every sequence of the equilibrium levels of a public good converges to zero through Milleron's (1972) replication. 
Several allocation rules that have been studied in mechanism design theory satisfy AUCR, including the efficient public goods provision rules, such as the Lindahl and the Clarke mechanisms. Our result implies that if the economy consists of many agents and each agent can choose whether or not to participate in an efficient public goods mechanism, then the equilibrium level of a public good is inefficient and very small.

Related to the above, we also discuss what it is to be an efficient public goods mechanism. An efficient public goods mechanism means that efficient public goods provision needs to be achieved for all subpopulation allocations. This is why when the population increases agents have more incentive to freeride. If the efficiency requirement for subpopulation allocations is dropped, then we can provide a more positive result. Consider the following rule.

Unanimous rule. Let $\varphi^{U}=\left(\varphi_{G}^{U}, \varphi_{\mathcal{T}}^{U}\right)$ be a unanimous rule: for all $e=$ $(\nu, C) \in \mathcal{E}$ and all $\widetilde{\nu} \leq \nu$,

$$
\varphi_{G}^{U}[\widetilde{\nu} ; \nu, C]=\left\{\begin{array}{ll}
\widetilde{g} & \text { if } \widetilde{\nu}=\nu \\
0 & \text { otherwise }
\end{array},\right.
$$

and

$$
u_{i}\left(\varphi_{G}^{U}\left[\nu^{\prime} ; \nu, C\right]\right)-\varphi_{\mathcal{T}}^{U}\left[\nu^{\prime} ; \nu, C\right]\left(u_{i}\right) \geq 0 \text { for all } u_{i} \in \operatorname{Supp}\left(\nu^{\prime}\right)
$$

Under this rule, a positive amount of a public good is produced if and only if all agents participate in public goods provision. The last condition is the individual rationality condition. Obviously, all agents participate at a Nash equilibrium even if the economy is replicated. Thus, there is a sequence of the levels of a public good at equilibrium that does not diminish to zero: even the efficient public goods provision can be achieved in an equilibrium sequence by setting $\widetilde{g}=\varphi_{G}^{E}[\nu ; \nu, C]$. Moreover, this rule assigns the same public goods provision level for all replications by setting $\varphi_{G}^{U}\left[\nu^{r} ; \nu^{r}, C\right]=\widetilde{g}$ for all $r=1,2, \ldots$ (replication invariance). However, since $\varphi^{U}$ has a discontinuity, it does not satisfy AUCR. This unanimity rule shows that even if a rule satisfies many properties, if AUCR is violated then the public goods provision level may not converge to zero.

Finally, we make some remarks on mixed strategy Nash equilibria. In this paper, we concentrated on pure strategy Nash equilibrium. One may wonder whether we can apply the same argument to mixed strategy Nash equilib- 
rium. ${ }^{15}$ It turns out to be difficult to check whether or not our approach can show the same result. Consider an economy $(\nu, C)$ with homogeneous agents (there is $u_{i} \in \mathcal{U}$ such that $\left.\operatorname{Supp}(\nu)=\left\{u_{i}\right\}\right)$. Let $\left|\nu\left(u_{i}\right)\right|=n$. Let us focus on a symmetric mixed strategy equilibrium with participation probability $p$. Then, there are $n+1$ possible states of realization. The equilibrium condition is

$$
\begin{aligned}
& \sum_{m=0}^{n-1} C(n-1, m) p^{m}(1-p)^{n-m-1}\left[u_{i}\left(\varphi_{G}[m+1 ; \nu, C]\right)-\varphi_{\mathcal{T}}[m+1 ; \nu, C]\left(u_{i}\right)\right] \\
= & \sum_{m=0}^{n-1} C(n-1, m) p^{m}(1-p)^{n-m-1} u_{i}\left(\varphi_{G}[m ; \nu, C]\right),
\end{aligned}
$$

or equivalently

$$
\begin{array}{r}
\sum_{m=0}^{n-1} C(n-1, m) p^{m}(1-p)^{n-m-1}\left[u_{i}\left(\varphi_{G}[m+1 ; \nu, C]\right)-u_{i}\left(\varphi_{G}[m ; \nu, C]\right)\right. \\
\left.-\varphi_{\mathcal{T}}[m+1 ; \nu, C]\left(u_{i}\right)\right]=0,
\end{array}
$$

where $\varphi_{G}[k ; \nu, C]$ and $\varphi_{\mathcal{T}}[k ; \nu, C]\left(u_{i}\right)$ denote the level of the public good and the payment when $k$ agents participate. However, this equation does not entail that for all realizations, $u_{i}\left(\varphi_{G}[m+1 ; \nu, C]\right)-u_{i}\left(\varphi_{G}[m ; \nu, C]\right)=(\geq$ ) $\varphi_{\mathcal{T}}[m ; \nu, C]\left(u_{i}\right)$ holds. We cannot obtain the condition in Lemma 1 even in the homogeneous agent case. Thus, it is not straightforward to extend our analysis to the case with mixed strategies.

\section{Appendix}

All proofs are collected in this appendix.

\footnotetext{
${ }^{15}$ Palfrey and Rosenthal (1984), Dixit and Oslon (2000), and Koriyama (2010) examine a mixed strategy Nash equilibrium of voluntary participation game. However, they do not investigate how Milleron's replication affects a mixed strategy Nash equilibrium.
} 


\section{Proof of Lemma 1}

By using condition (i) of the necessary and sufficient condition for Nash equilibrium, we have

$$
\begin{aligned}
& \sum_{u_{i} \in \operatorname{Supp}(\nu)} \mu[s]\left(u_{i}\right) u_{i}\left(\varphi_{G}[\mu[s] ; \nu, C]\right)-\sum_{u_{i} \in \operatorname{Supp}(\nu)} \mu[s]\left(u_{i}\right) \varphi_{\mathcal{T}}[\mu[s] ; \nu, C]\left(u_{i}\right) \\
\geq & \sum_{u_{i} \in \operatorname{Supp}(\nu)} \mu[s]\left(u_{i}\right) u_{i}\left(\varphi_{G}\left[\mu_{(i,-1)}[s] ; \nu, C\right]\right),
\end{aligned}
$$

or

$$
\begin{aligned}
& \sum_{u_{i} \in \operatorname{Supp}(\nu)} \mu[s]\left(u_{i}\right) u_{i}\left(\varphi_{G}[\mu[s] ; \nu, C]\right)-\sum_{u_{i} \in \operatorname{Supp}(\nu)} \mu[s]\left(u_{i}\right) u_{i}\left(\varphi_{G}\left[\mu_{(i,-1)}[s] ; \nu, C\right]\right) \\
\geq & \sum_{u_{i} \in \operatorname{Supp}(\nu)} \mu[s]\left(u_{i}\right) \varphi_{\mathcal{T}}[\mu[s] ; \nu, C]\left(u_{i}\right) \\
\geq & C\left(\varphi_{G}[\mu[s] ; \nu, C]\right),
\end{aligned}
$$

where the last inequality holds by the feasibility constraint.

\section{Proof of Lemma 2}

We first show Claims 1 and 2 and then we show Lemma 2 by using these claims.

Claim 1 Let $r \in \mathbb{Z}_{++}$and let $s_{r} \in N E\left(\Gamma\left(\nu^{r}, C, \phi\right)\right)$ be such that $\left|\mu\left[s_{r}\right]\right| \neq 0$. Since the slope of utility function is bounded above by some $M>0$, we have

$$
M \sum_{u_{i}^{r} \in \operatorname{Supp}(\nu)}\left(\varphi_{G}\left[\mu\left[s_{r}\right] ; \nu^{r}, C\right]-\varphi_{G}\left[\mu_{\left(i^{r},-1\right)}\left[s_{r}\right] ; \nu^{r}, C\right]\right) \geq \frac{C\left(\varphi_{G}\left[\mu\left[s_{r}\right] ; \nu^{r}, C\right]\right)}{|\nu|} .
$$

Proof of Claim 1. Since $s_{r}$ is a Nash equilibrium for all $r$, by Lemma 1 we have

$$
\begin{aligned}
& \sum_{u_{i}^{r} \in \operatorname{Supp}\left(\nu^{r}\right)} \mu\left[s_{r}\right]\left(u_{i}^{r}\right)\left[u_{i}^{r}\left(\varphi_{G}\left[\mu\left[s_{r}\right] ; \nu^{r}, C\right]\right)-u_{i}^{r}\left(\varphi_{G}\left[\mu_{\left(i^{r},-1\right)}\left[s_{r}\right] ; \nu^{r}, C\right]\right)\right] \\
\geq & C\left(\varphi_{G}\left[\mu\left[s_{r}\right] ; \nu^{r}, C\right]\right),
\end{aligned}
$$


if $\left|\mu\left[s_{r}\right]\right| \neq 0$. Since $\frac{u_{i}^{r}\left(g_{1}\right)-u_{i}^{r}\left(g_{2}\right)}{g_{1}-g_{2}} \leq \frac{u_{i}\left(g_{1}\right)-u_{i}\left(g_{2}\right)}{r\left(g_{1}-g_{2}\right)} \leq \frac{M}{r}$ for all $u_{i}$ and all $g_{1}, g_{2} \geq 0$,

$$
\begin{aligned}
& \sum_{u_{i}^{r} \in \operatorname{Supp}\left(\nu^{r}\right)} \mu\left[s_{r}\right]\left(u_{i}^{r}\right)\left[u_{i}^{r}\left(\varphi_{G}\left[\mu\left[s_{r}\right] ; \nu^{r}, C\right]\right)-u_{i}^{r}\left(\varphi_{G}\left[\mu_{\left(i^{r},-1\right)}\left[s_{r}\right] ; \nu^{r}, C\right]\right)\right] \\
\leq & M \sum_{u_{i}^{r} \in \operatorname{Supp}\left(\nu^{r}\right)} \frac{\mu\left[s_{r}\right]\left(u_{i}^{r}\right)}{r}\left(\varphi_{G}\left[\mu\left[s_{r}\right] ; \nu^{r}, C\right]-\varphi_{G}\left[\mu_{\left(i^{r},-1\right)}\left[s_{r}\right] ; \nu^{r}, C\right]\right)
\end{aligned}
$$

Thus,

$$
\begin{aligned}
& M \sum_{\substack{u_{i}^{r} \in \operatorname{Supp}\left(\nu^{r}\right) \\
\geq}} \frac{\mu\left[s_{r}\right]\left(u_{i}^{r}\right)}{r}\left(\varphi_{G}\left[\mu\left[s_{r}\right] ; \nu^{r}, C\right]-\varphi_{G}\left[\mu_{\left(i^{r},-1\right)}\left[s_{r}\right] ; \nu^{r}, C\right]\right) \\
& C\left(\varphi_{G}\left[\mu\left[s_{r}\right] ; \nu^{r}, C\right]\right) .
\end{aligned}
$$

Dividing both sides of (3) by $|\nu|>0$, we have

$$
M \sum_{u_{i}^{r} \in \operatorname{Supp}\left(\nu^{r}\right)} \frac{\mu\left[s_{r}\right]\left(u_{i}^{r}\right)}{r|\nu|}\left(\varphi_{G}\left[\mu\left[s_{r}\right] ; \nu^{r}, C\right]-\varphi_{G}\left[\mu_{\left(i^{r},-1\right)}\left[s_{r}\right] ; \nu^{r}, C\right]\right) \geq \frac{C\left(\varphi_{G}\left[\mu\left[s_{r}\right] ; \nu^{r}, C\right]\right)}{|\nu|} .
$$

Since $r|\nu| \geq \mu\left[s_{r}\right]\left(u_{i}^{r}\right)$ for all $u_{i}^{r} \in \operatorname{Supp}\left(\nu^{r}\right)$

$$
\begin{aligned}
& M \sum_{u_{i}^{r} \in \operatorname{Supp}\left(\nu^{r}\right)} \frac{\mu\left[s_{r}\right]\left(u_{i}^{r}\right)}{r|\nu|}\left(\varphi_{G}\left[\mu\left[s_{r}\right] ; \nu^{r}, C\right]-\varphi_{G}\left[\mu_{\left(i^{r},-1\right)}\left[s_{r}\right] ; \nu^{r}, C\right]\right) \\
\leq & M \sum_{u_{i}^{r} \in \operatorname{Supp}(\nu)}\left(\varphi_{G}\left[\mu\left[s_{r}\right] ; \nu^{r}, C\right]-\varphi_{G}\left[\mu_{\left(i^{r},-1\right)}\left[s_{r}\right] ; \nu^{r}, C\right]\right) .
\end{aligned}
$$

Therefore, we have (2).

Claim 2 Let $\left\{s_{r}\right\}$ be an infinite sequence of Nash equilibria. Then, for any $\epsilon>0$, there is $r_{\epsilon} \in \mathbb{R}_{++}$such that $\varphi_{G}\left[\mu\left[s_{r}\right] ; \nu^{r}, C\right]-\varphi_{G}\left[\mu_{\left(i^{r},-1\right)}\left[s_{r}\right] ; \nu^{r}, C\right]<\epsilon$ for any $r \geq r_{\epsilon}$ and all $u_{i}^{r} \in \operatorname{Supp}\left(\nu^{r}\right)$ on the sequence, if $\varphi$ satisfies $A U C R$.

Proof of Claim 2. For all $s_{r}$ on the sequence and all $u_{i}^{r} \in \operatorname{Supp}\left(\nu^{r}\right)$, we have $\frac{\left|\mu\left[s_{r}\right]-\mu_{\left(i^{r},-1\right)}\left[s_{r}\right]\right|}{\left|\nu^{r}\right|}=\frac{1}{r|\nu|}$. Hence, there exists $r_{\epsilon}>0$ such that $\frac{1}{r|\nu|} \leq \delta$ for all $r \geq r_{\epsilon}$, which implies that, for any $\epsilon>0$, there is $r_{\epsilon}>0$ such that $\left|\varphi_{G}\left[\mu\left[s_{r}\right] ; \nu^{r}, C\right]-\varphi_{G}\left[\mu_{\left(i^{r},-1\right)}\left[s_{r}\right] ; \nu^{r}, C\right]\right|<\epsilon$ for any $r \geq r_{\epsilon}$ and all $u_{i}^{r} \in \operatorname{Supp}\left(\nu^{r}\right)$ by AUCR. Since $\mu\left[s_{r}\right]$ is a Nash equilibrium participation, 
the content of $|\cdot|$ is positive.

Proof of Lemma 2. Suppose, on the contrary, that there is some positive public good provision level $g>0$ such that for all $\bar{r}>0$, there exist $r \geq \bar{r}$ and a Nash equilibrium $s_{r}$ providing $g$ or more units of the public good. When $\left|\mu\left[s_{r}\right]\right|=0, \varphi_{G}\left[\mu\left[s_{r}\right] ; \nu^{r}, C\right]=0$ holds and the above supposition does not hold. So, we have $\left|\mu\left[s_{r}\right]\right| \neq 0$ for all $r \geq \bar{r}$. From this, we can construct an infinite (sub)sequence of Nash equilibria $\left\{s_{r}\right\}$ such that $\varphi_{G}\left[\mu\left[s_{r}\right] ; \nu^{r}, C\right] \geq g$ in each $s_{r}$ of this sequence.

From Claim 2, $\left|\operatorname{Supp}\left(\nu^{r}\right)\right|=|\operatorname{Supp}(\nu)|<\infty$, and the finiteness of $M$, the left-hand side of (2) diminishes to zero as $r$ goes to infinity. However, since $C$ is nondecreasing and $C(g)>0$ for all $g>0$, we have

$$
\frac{C\left(\varphi_{G}\left[\mu\left[s_{r}\right] ; \nu^{r}, C\right]\right)}{|\nu|} \geq \frac{C(g)}{|\nu|}>0,
$$

which indicates that $s_{r}$ cannot be a Nash equilibrium of $\Gamma\left(\nu^{r}, C, \varphi\right)$ for sufficiently large $r$ unless $g=0$ by (2), since $|\nu|$ is the population of the original economy and is fixed. This is a contradiction.

\section{Proof of Theorem 1}

Suppose not: for some $(\nu, C)$, there exist $\bar{g}>0$ and a (sub)sequence of Nash equilibria, $\left\{s_{r}\right\}_{r=1}^{\infty}$, such that, for all $r=1,2, \ldots, s_{r} \in N E\left(\Gamma\left(\nu^{r}, C, \varphi\right)\right)$ and $\varphi_{G}\left[\mu\left[s_{r}\right] ; \nu^{r}, C\right] \geq \bar{g}$. However, it is clear from Lemma 2 that Nashequilibrium sequences cannot be constructed in such a way that $s_{r}$ supports the provision of not less than $\bar{g}$ units of the public good for any $r=1,2, \ldots$. This is a contradiction.

\section{Proof of Proposition 1}

First note that under the domain restriction, $\varphi_{G}^{E}$ is nonempty-valued. ${ }^{16}$ Let $(\nu, C) \in \mathcal{E}$. For all $\delta>0$ and all $r \in \mathbb{Z}_{++}$, let $k(r ; \delta, \nu) \equiv \delta r|\nu|$ and let $k(r ; \delta, \nu)$ be the largest integer $k$ such that $k \leq k(r ; \delta, \nu)$. By the Samuel$\overline{\text { son rule }}, \varphi_{G}^{E}$ satisfies $\sum_{u \in S u p p(\hat{\nu})} \hat{\nu}(u) u^{\prime}\left(\varphi_{G}^{E}[\hat{\nu} ; \nu, C]\right) \leq C^{\prime}\left(\varphi_{G}^{E}[\hat{\nu} ; \nu, C]\right)$ for all $e=(\nu, C) \in \mathcal{E}$ and all $\hat{\nu} \leq \nu$. If the condition is not satisfied for $(\nu ; \nu, C)$ with equality, $\varphi_{G}^{E}[\nu ; \nu, C]=0$ and $\varphi_{G}^{E}\left[\nu^{r} ; \nu^{r}, C\right]=0$ hold, so we

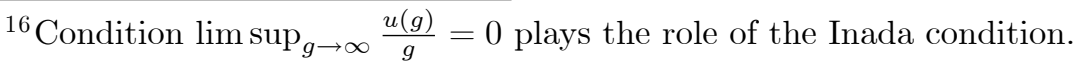


concentrate the case for $\varphi_{G}^{E}[\nu ; \nu, C]>0$. Let $\bar{u}^{\prime}[\nu]: \mathbb{R}_{++} \rightarrow \mathbb{R}_{+}$such that $\bar{u}^{\prime}[\nu](g) \equiv \max _{u \in \operatorname{Supp}(\nu)} u^{\prime}(g)$ for all $g \in \mathbb{R}_{+}$. This function $\bar{u}^{\prime}[\nu]$ is nonincreasing, satisfying $\bar{u}^{\prime}[\nu](g) \geq u^{\prime}(g)$ for all $u \in \operatorname{Supp}(\nu)$. Let $\underline{\varphi_{G}}[\hat{\nu}, k ; \nu, C]$ be the lower bound for the public good provisions level when $k$ agents leave group $\hat{\nu}$ : i.e., $\varphi_{G}[\hat{\nu}, k ; \nu, C]$ is such that

$$
\sum_{u \in \operatorname{Supp}(\hat{\nu})} \hat{\nu}(u) u^{\prime}\left(\underline{\varphi_{G}}[\hat{\nu}, k ; \nu, C]\right)-k \bar{u}^{\prime}[\nu]\left(\underline{\varphi_{G}}[\hat{\nu}, k ; \nu, C]\right)=C^{\prime}\left(\underline{\varphi_{G}}[\hat{\nu}, k ; \nu, C]\right),
$$

if there is a solution to the above equation, and $\underline{\varphi_{G}}[\hat{\nu}, k ; \nu, C]=0$, otherwise.

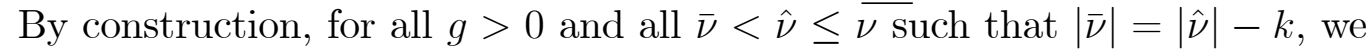
have

$$
\sum_{u \in \operatorname{Supp}(\hat{\nu})} \hat{\nu}(u) u^{\prime}(g)-k \bar{u}^{\prime}[\nu](g) \leq \sum_{u \in \operatorname{Supp}(\hat{\nu})} \bar{\nu}(u) u^{\prime}(g) .
$$

Recall that $u$ and $C$ are continuously differentiable, $u^{\prime}$ is non-increasing, and $C^{\prime}$ is nondecreasing in $g$. Thus, if there is a solution in (4), it must be unique, and it satisfies $\varphi_{G}[\hat{\nu}, k ; \nu, C] \leq \varphi_{G}^{E}[\bar{\nu} ; \nu, C]$ for all $\bar{\nu}<\hat{\nu} \leq \nu$ such that $|\bar{\nu}|=|\hat{\nu}|-k$. If there is no solution in $(4), \underline{\varphi_{G}}[\hat{\nu}, k ; \nu, C]=0$ and $\underline{\varphi_{G}}[\hat{\nu}, k ; \nu, C] \leq \varphi_{G}^{E}[\bar{\nu} ; \nu, C]$ is again satisfied.

Let $\overline{\Delta G(k ; \nu, C)} \equiv \max _{\hat{\nu} \leq \nu}\left|\varphi_{G}^{E}[\hat{\nu} ; \nu, C]-\underline{\varphi_{G}}[\hat{\nu}, k ; \nu, C]\right|:$ i.e., the maximum possible reduction of the public goods provision level in all possible public goods provision groups $\hat{\nu}$ of $(\nu, C)$ by $k$ people leaving group $\hat{\nu}$. To go one step further, let us extend this notion to a possibly continuous $\hat{\nu}$ case (by naturally extending $\varphi_{G}[\hat{\nu}, k ; \nu, C]$ for all real numbers $\left.k\right)$. We now allow for any (nonnegative) real-valued function $\hat{\nu}: \mathcal{U} \rightarrow \mathbb{R}_{+}$such that $\hat{\nu}(u) \leq \nu(u)$ for all $u \in \mathcal{U}$. Since $\varphi_{G}^{E}[\hat{\nu} ; \nu, C]$ and $\varphi_{G}[\hat{\nu}, k ; \nu, C]$ are continuous in $\hat{\nu}$ and $\hat{\nu}$ satisfies $0 \leq \hat{\nu} \leq \nu, \varphi_{G}[\hat{\nu}, k ; \nu, C]$ and $\overline{\Delta G(k ; \nu, C)}$ are still well defined.

Now, we apply the above concept to replica economies of $(\nu, C)$. For all $\widetilde{\nu}^{r}, \bar{\nu}^{r} \in \mathcal{N}$ such that $\widetilde{\nu}^{r} \leq \bar{\nu}^{r} \leq \nu^{r}$ with $\left|\bar{\nu}^{r}-\widetilde{\nu}^{r}\right| \leq k \underline{k(r ; \delta, \nu)}$, we have

$$
\begin{aligned}
\left|\varphi_{G}^{E}\left[\bar{\nu} ; \nu^{r}, C\right]-\varphi_{G}^{E}\left[\widetilde{\nu} ; \nu^{r}, C\right]\right| & \leq \overline{\Delta G\left(k(r ; \delta, \nu) ; \nu^{r}, C\right)} \\
& \leq \overline{\Delta G\left(k(r ; \delta, \nu) ; \nu^{r}, C\right)} \\
& =\overline{\Delta G\left(\delta r|\nu| ; \nu^{r}, C\right)} \\
& =\overline{\Delta G(\delta|\nu| ; \nu, C)} .
\end{aligned}
$$

The equalities hold by the definitions of $k(r ; \delta, \nu)$ and the Milleron's replication, respectively. Clearly, if $\delta=0$, then $\overline{\Delta G(\delta|\nu| ; \nu, C)}=0$ and if $\delta \geq 1$, 
then $\overline{\Delta G(\delta|\nu| ; \nu, C)}=\varphi_{G}^{E}[\nu ; \nu, C]$ by the efficiency of $\varphi_{G}^{E}$. If $0 \leq \delta \leq 1$,

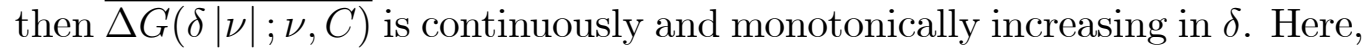
continuity of $\overline{\Delta G(\delta|\nu| ; \nu, C)}$ is assured by nonincreasingness of $u^{\prime}$ and nondecreasingness of $C^{\prime}$, since it is a solution of (4).

Let $\epsilon>0$. It is trivial if $\epsilon \geq \varphi_{G}^{E}[\nu ; \nu, C]$. Otherwise, let $\delta_{\epsilon}$ be defined by $\epsilon=\overline{\Delta G\left(\delta_{\epsilon}|\nu| ; \nu, C\right)}$. Take $\bar{\delta}_{\epsilon}$ such that $0<\bar{\delta}_{\epsilon}<\delta_{\epsilon}$ and take an arbitrary $\bar{r} \in \mathbb{Z}_{++}$. Then, for any pair $\bar{\nu}, \widetilde{\nu}$ and all $r \in \mathbb{Z}_{++}$such that $r \geq \bar{r}, \frac{|\widetilde{\nu}-\bar{\nu}|}{\left|\nu^{r}\right|} \leq \bar{\delta}_{\epsilon}$, $\left|\varphi_{G}^{E}\left[\bar{\nu}^{r} ; \nu^{r}, C\right]-\varphi_{G}^{E}\left[\widetilde{\nu}^{r} ; \nu^{r}, C\right]\right|<\epsilon$.

Therefore, $\varphi_{G}^{E}$ satisfies AUCR. $\square$

\section{Proof of Proposition 2}

Let $(\nu, C) \in \mathcal{E}$. Let $r \in \mathbb{Z}_{++}$. Since $\varphi_{G}^{F}$ is a fixed contribution rule with budget balance,

$$
\sum_{u_{i}^{r} \in \operatorname{Supp}\left(\nu^{r}\right)} \nu^{r}\left(u_{i}^{r}\right) \varphi_{\mathcal{T}}^{F}\left[\nu^{r} ; \nu^{r}, C\right]\left(u_{i}^{r}\right)=C\left(\varphi_{G}^{F}\left[\nu^{r} ; \nu^{r}, C\right]\right) \leq C(\bar{G}(\nu)) .
$$

Thus, for all $u_{i}^{r} \in \operatorname{Supp}\left(\nu^{r}\right)$,

$$
\varphi_{\mathcal{T}}^{F}\left[\nu^{r} ; \nu^{r}, C\right]\left(u_{i}^{r}\right) \leq \frac{1}{\nu^{r}\left(u_{i}^{r}\right)} C(\bar{G}(\nu)) \leq \frac{1}{r \underline{\nu}} C(\bar{G}(\nu)),
$$

where $\underline{\nu}=\min _{u_{i} \in \operatorname{Supp}(\nu)} \nu\left(u_{i}\right)$. For all $\delta>0$, all $r \in \mathbb{Z}_{++}$, and all $\widetilde{\nu}, \bar{\nu} \in \mathcal{N}$ such that $\bar{\nu} \leq \widetilde{\nu} \leq \nu^{r}$ and $\frac{|\widetilde{\nu}-\bar{\nu}|}{\left|\nu^{r}\right|} \leq \delta$,

$$
\begin{aligned}
& \sum_{u^{r} \in \operatorname{Supp}\left(\nu^{r}\right)} \widetilde{\nu}\left(u^{r}\right) \varphi_{\mathcal{T}}^{F}\left[\widetilde{\nu} ; \nu^{r}, C\right]\left(u^{r}\right)-\sum_{u^{r} \in \operatorname{Supp}\left(\nu^{r}\right)} \bar{\nu}\left(u^{r}\right) \varphi_{\mathcal{T}}^{F}\left[\bar{\nu} ; \nu^{r}, C\right]\left(u^{r}\right) \\
\leq & |\widetilde{\nu}-\bar{\nu}| \times \frac{1}{r \underline{\nu}} C(\bar{G}(\nu)) \\
= & \frac{|\widetilde{\nu}-\bar{\nu}|}{\left|\nu^{r}\right|} \times \frac{|\nu|}{\underline{\nu}} \times C(\bar{G}(\nu)) \\
\leq & \delta \times \frac{|\nu|}{\underline{\nu}} \times C(\bar{G}(\nu))
\end{aligned}
$$

Note that $\delta \times \frac{|\nu|}{\underline{\nu}} \times C(\bar{G}(\nu))$ is independent of $r$.

Take any pair $\widetilde{\nu}, \bar{\nu}$ such that $\bar{\nu} \leq \widetilde{\nu} \leq \nu^{r}$ and $\frac{|\widetilde{\nu}-\bar{\nu}|}{\left|\nu^{r}\right|} \leq \delta$. Suppose that $\sum_{u^{r} \in \operatorname{Supp}\left(\widetilde{\nu}^{r}\right)} \widetilde{\nu}\left(u^{r}\right) \varphi_{\mathcal{T}}^{F}\left[\widetilde{\nu}^{r} ; \nu^{r}, C\right]\left(u^{r}\right)=0$. Then, by the definition of fixed contribution rule, $\sum_{u^{r} \in \operatorname{Supp}\left(\bar{\nu}^{r}\right)} \bar{\nu}\left(u^{r}\right) \varphi_{\mathcal{T}}^{F}\left[\bar{\nu}^{r} ; \nu^{r}, C\right]\left(u^{r}\right)=0$ as well. So, this case 
AUCR is trivially satisfied. If $\varphi_{\mathcal{T}}^{F}\left[\widetilde{\nu} ; \nu^{r}, C\right]\left(u^{r}\right)>0$ for some $u^{r} \in \operatorname{Supp}(\widetilde{\nu})$, we have $\sum_{u^{r} \in \operatorname{Supp}\left(\nu^{r}\right)} \widetilde{\nu}\left(u^{r}\right) \varphi_{\mathcal{T}}^{F}\left[\widetilde{\nu} ; \nu^{r}, C\right]\left(u^{r}\right) \geq \sum_{u^{r} \in \operatorname{Supp}\left(\nu^{r}\right)} \bar{\nu}\left(u^{r}\right) \varphi_{\mathcal{T}}^{F}\left[\bar{\nu} ; \nu^{r}, C\right]\left(u^{r}\right)$. Note that we have $C^{-1 \prime}(g)<\frac{1}{c}$ for all $g \geq 0$, since $C^{\prime}(g)>\underline{c}>0$ for all $g \geq 0$. Thus, we have

$$
\begin{aligned}
& \varphi_{G}^{F}\left[\widetilde{\nu} ; \nu^{r}, C\right]-\varphi_{G}^{F}\left[\bar{\nu} ; \nu^{r}, C\right] \\
& =C^{-1}\left(\sum_{u^{r} \in \operatorname{Supp}\left(\nu^{r}\right)} \widetilde{\nu}\left(u^{r}\right) \varphi_{\mathcal{T}}^{F}\left[\widetilde{\nu} ; \nu^{r}, C\right]\left(u^{r}\right)\right)-C^{-1}\left(\sum_{u^{r} \in \operatorname{Supp}\left(\nu^{r}\right)} \bar{\nu}\left(u^{r}\right) \varphi_{\mathcal{T}}^{F}\left[\bar{\nu} ; \nu^{r}, C\right]\left(u^{r}\right)\right)
\end{aligned}
$$

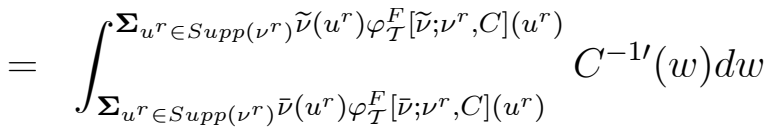

$$
\begin{aligned}
& <\int_{\boldsymbol{\Sigma}_{u^{r} \in \operatorname{Supp}\left(\nu^{r}\right)} \bar{\nu}\left(u^{r}\right) \varphi_{\mathcal{T}}^{F}\left[\bar{\nu} ; \nu^{r}, C\right]\left(u^{r}\right)} \frac{\underline{c}}{\boldsymbol{\Sigma}_{u^{r} \in \operatorname{Supp}\left(\nu^{r}\right) \widetilde{\nu}\left(u^{r}\right) \varphi_{\mathcal{T}}^{F}\left[\widetilde{\nu} ; \nu^{r}, C\right]\left(u^{r}\right)}} d w \\
& =\frac{1}{\underline{c}}\left(\sum_{u^{r} \in \operatorname{Supp}\left(\nu^{r}\right)} \widetilde{\nu}\left(u^{r}\right) \varphi_{\mathcal{T}}^{F}\left[\widetilde{\nu} ; \nu^{r}, C\right]\left(u^{r}\right)-\sum_{u^{r} \in \operatorname{Supp}\left(\nu^{r}\right)} \bar{\nu}\left(u^{r}\right) \varphi_{\mathcal{T}}^{F}\left[\bar{\nu} ; \nu^{r}, C\right]\left(u^{r}\right)\right) \\
& \leq \frac{1}{\underline{c}} \times \delta \times \frac{|\nu|}{\underline{\nu}} \times C(\bar{G}(\nu)) . \quad(\text { by }(5))
\end{aligned}
$$

Therefore, for all $\delta>0$ and for all $r \in \mathbb{Z}_{++}$and all $\widetilde{\nu}, \bar{\nu} \in \mathcal{N}$ such that $\bar{\nu} \leq \widetilde{\nu} \leq \nu^{r}$ and $\frac{|\widetilde{\nu}-\bar{\nu}|}{\left|\nu^{r}\right|} \leq \delta$,

$$
\varphi_{G}^{F}\left[\widetilde{\nu} ; \nu^{r}, C\right]-\varphi_{G}^{F}\left[\bar{\nu} ; \nu^{r}, C\right] \leq \frac{\delta|\nu|}{\underline{c \nu}} \times C(\bar{G}(\nu)) .
$$

If $\delta \in \mathbb{R}_{++}$, the RHS of (6) is positive and finite. They converge to zero as $\delta$ goes to zero. Therefore, by (6), for any $\epsilon>0$, we can find $\delta_{\epsilon}=$ $\frac{c \nu}{C(\bar{G}(\nu))|\nu|}(0.9 \epsilon)>0$ and $\bar{r} \in \mathbb{Z}_{++}$such that for all $r \geq \bar{r}$ and all $\widetilde{\nu}^{r}, \bar{\nu}^{r} \in \mathcal{N}$ such that $\bar{\nu}^{r} \leq \widetilde{\nu}^{r} \leq \nu^{r}$ and $\frac{\left|\widetilde{\nu}^{r}-\bar{\nu}^{r}\right|}{\left|\nu^{r}\right|} \leq \delta_{\epsilon}, \varphi_{G}^{F}\left[\widetilde{\nu}^{r} ; \nu^{r}, C\right]-\varphi_{G}^{F}\left[\bar{\nu}^{r} ; \nu^{r}, C\right]<\epsilon$.

\section{Proof of Lemma 3}

Under the assumption of $C$, the inverse function of $C, C^{-1}$, exists. Then, $i \in N^{\nu}$ 's payoff is rewritten as $u_{i}\left(C^{-1}\left(\sum_{j \in N_{\tilde{\nu}}} x_{j}\right)\right)-x_{i}$.

We first show that for all $u \in S u p p(\nu), \arg \max _{x \geq 0} u\left(C^{-1}(x)\right)-x$ is not empty. By the assumption of $u$ and $C, u\left(C^{-1}(x)\right)$ is increasing and strictly 
concave in $x$. Thus, $u^{\prime}\left(C^{-1}(x)\right) \cdot C^{-1 \prime}(x)$ is decreasing in $x$. For all $u \in$ $\operatorname{Supp}(\nu)$ such that $u^{\prime}(0) \cdot C^{-1 \prime}(0) \leq 1$, then $\arg \max _{x \geq 0} u\left(C^{-1}(x)\right)-x=\{0\}$. Consider $u \in \operatorname{Supp}(\nu)$ such that $u^{\prime}(0) \cdot C^{-1 \prime}(0)>1$. Since the slope of $u$ is uniformly bounded above by $M<\infty$ for all $u \in \operatorname{Supp}(\nu), u^{\prime}(g) \leq M$ for all $u \in \operatorname{Supp}(\nu)$ and all $g \in \mathbb{R}_{+}$. By the Inada condition (iii), there exists $x \in \mathbb{R}_{+}$ such that $u_{i}^{\prime}\left(C^{-1}(x)\right) \cdot C^{-1 \prime}(x)=1$, since $\frac{d u\left(C^{-1}(x)\right)}{d x}=u^{\prime}\left(C^{-1}(x)\right) \cdot C^{-1 \prime}(x)$ is continuous and decreasing in $x$, which implies that arg $\max _{x>0} u\left(C^{-1}(x)\right)-x$ is not empty.

We now characterize the best response function for all agents. Since $u_{i}^{\prime}\left(C^{-1}(x)\right) \cdot C^{-1 \prime}(x)$ is decreasing in $x$, then for all $i \in N^{\nu}$ with $u \in \operatorname{Supp}(\nu)$, all $x \in \prod_{j \in N^{\nu}} X_{j}$, and all $g$ such that $g=C^{-1}\left(\sum_{j \in N^{\nu}} x_{j}\right), g<g_{i}^{*}$ if and only if $u_{i}^{\prime}(g) \cdot C^{-1 \prime}\left(\sum_{j \in N^{\nu}} x_{j}\right)>1$. Therefore, the best response function of $i \in N^{\nu}$ with $u \in \operatorname{Supp}(\nu), b_{i}: \prod_{j \neq i} X_{j} \rightarrow X_{i}$, is

$b_{i}\left(x_{-j}\right)= \begin{cases}0 & \text { if } g_{i}^{*} \leq C^{-1}\left(\sum_{j \neq i} x_{j}\right) \\ x_{i} \text { such that } g_{i}^{*}=C^{-1}\left(\sum_{j \neq i} x_{j}+x_{i}\right) & \text { otherwise. }\end{cases}$

By the best response function, the set of Nash equilibria is clearly characterized as (1).

\section{Proof of Proposition 3}

We show that for all $(\nu, C) \in \mathcal{N}$ and all $\epsilon>0$, there exists $r_{\epsilon} \in \mathbb{Z}_{++}$such that $\left|\varphi_{G}^{V C}\left[\widetilde{\nu} ; \nu^{r}, C\right]-\varphi_{G}^{V C}\left[\bar{\nu} ; \nu^{r}, C\right]\right|<\epsilon$ for all $r \geq r_{\epsilon}$ and all $\widetilde{\nu}, \bar{\nu}$ such that $\widetilde{\nu} \leq \bar{\nu} \leq \nu^{r}$ and $\frac{|\widetilde{\nu}-\bar{\nu}|}{\left|\nu^{r}\right|} \leq \frac{1}{r_{\epsilon}|\nu|}$. If so, we complete the proof by letting $\delta=\frac{1}{r_{\epsilon}|\nu|}$. Suppose not. Then, there is a sequence $\left\{\left(\widetilde{\nu}_{t(r)}, \bar{\nu}_{t(r)}\right)\right\}_{r=1}^{\infty}$ such that $t(r) \geq r$, $\frac{\left|\widetilde{\nu}_{t(r)}-\bar{\nu}_{t(r)}\right|}{\left|\nu^{t(r)}\right|} \leq \frac{1}{r|\nu|}$ and $\left|\varphi_{G}^{V C}\left[\widetilde{\nu}_{t(r)} ; \nu^{t(r)}, C\right]-\varphi_{G}^{V C}\left[\bar{\nu}_{t(r)} ; \nu^{t(r)}, C\right]\right| \geq \epsilon$ are satisfied for all $r \in \mathbb{Z}_{++}$. However, $g_{\widetilde{\nu}}^{\max }$ converges to zero as $r$ goes to infinity for all $\widetilde{\nu} \leq \nu^{r},{ }^{17}$ which implies that $\left|\varphi_{G}^{V C}\left[\widetilde{\nu}_{t(r)} ; \nu^{t(r)}, C\right]-\varphi_{G}^{V C}\left[\bar{\nu}_{t(r)} ; \nu^{t(r)}, C\right]\right| \geq \epsilon$ is impossible for sufficiently large $r$. This is a contradiction. $\square$

\footnotetext{
${ }^{17}$ We can check this from the first-order condition $\frac{1}{r} u^{\prime-1 \prime}(x)=1$, where $g=C^{-1}(x)$. Under our conditions for $u$ and $C$, there may be a case in which this equation does not hold for sufficiently large $r$. Then, the level of the public good is zero. In the other case, as $r$ increases, if the equation holds, then $x$ declines and goes to zero, which imples that the level of the public good goes to zero.
} 


\section{References}

[1] Allouch, N., and M. Wooders (2008) Price Taking Equilibrium in Economies with Multiple Memberships in Clubs and Unbounded Club Sizes, Journal of Economic Theory, Vol.140, pp. 246-278.

[2] Bergstrom, T., L. Blume, and H. Varian (1986) On the Private Provision of Public Goods, Journal of Public Economics, Vol.29, pp. 25-49.

[3] Clarke, E. (1971) Multi-part Pricing of Public Goods, Public Choice, Vol.11, pp. 17-33.

[4] Conley, J.P. (1994) Convergence Theorems on the Core of a Public Goods Economy: Sufficient Conditions, Journal of Economic Theory, Vol.62, pp. 161-185.

[5] Dixit, A., and M. Olson (2000) Does Voluntary Participation Undermine the Coase Theorem?, Journal of Public Economics, Vol.76, pp. 309-335.

[6] Furusawa, T., and H. Konishi (2011) Contributing or Free-Riding?: Voluntary Participation in a Public Good Economy, Theoretical Economics, Vol. 6, pp. 219-256.

[7] Groves T., and J. Ledyard (1977) Optimal Allocation of Public Goods: A Solution to the "Free Rider" Problem, Econometrica, Vol.45, pp. 783811.

[8] Healy, P.J. (2010) Equilibrium Participation in Public Goods Allocations, Review of Economic Design, Vol.14, pp. 27-50.

[9] Hurwicz, L. (1979) Outcome Functions Yielding Walrasian and Lindahl Allocations at Nash Equilibrium Points, Review of Economic Studies, Vol.46, pp. 217-225.

[10] Koriyama, Y. (2010) Freedom to Not Join: A Voluntary Participation Game of Discrete Public Good Provision, mimeo.

[11] Milleron, J.C. (1972) Theory of Value with Public Goods: A Survey Article, Journal of Economic Theory, Vol.5, pp. 419-477. 
[12] Muench, T.J. (1972) The Core and the Lindahl Equilibrium of an Economy with a Public Good: An Example, Journal of Economic Theory, Vol.4, pp. 241-255.

[13] Palfrey, R.T., and H. Rosenthal (1984) Participation and the Provision of Discrete Public Goods: A Strategic Analysis, Journal of Public Economics, Vol.24, pp. 171-193.

[14] Saijo, T., and T. Yamato (1999) A Voluntary Participation Game with a Non-Excludable Public Good, Journal of Economic Theory Vol.84, pp. 227-242.

[15] Saijo, T., and T. Yamato (2010) Fundamental Impossibility Theorems on Voluntary Participation in the Provision of Non-excludable Public Goods, Review of Economic Design, Vol.14, pp. 51-73.

[16] Samuelson, P.A. (1954) The Pure Theory of Public Expenditure, Review of Economics and Statistics, Vol.36, pp. 387-389.

[17] Shinohara, R. (2009) The Possibility of Efficient Provision of a Public Good in Voluntary Participation Games, Social Choice and Welfare, Vol.32, pp. 367-387.

[18] Shinohara, R. (2010) Coalition-proof Equilibria in a Voluntary Participation Game, International Journal of Game Theory, Vol. 39, pp. 603615 .

[19] Walker, M. (1981) A Simple Incentive Compatible Scheme for Attaining Lindahl Allocations, Econometrica, Vol.49, pp. 65-71.

[20] Wooders, M. (1983) The Epsilon Core of a Large Replica Game, Journal of Mathematical Economics, Vol.11, pp. 277-300.

[21] Wooders, M. (1989) A Tiebout Theorem, Mathematical Social Sciences, Vol.18, Issue 1, August 1989, Pages 33-55

[22] Wooders, M. (2008) Small Group Effectiveness, Per Capita Boundedness, and Nonemptiness of Approximate Cores, Journal of Mathematical Economics, Vol.44, pp. 888-906. 